\title{
Stroboscopic Vision as a Dynamic Sensory Reweighting Alternative to the Sensory Organization Test
}

\author{
Kyung-Min Kim, Joo-Sung Kim, Jeonghoon Oh, and Dustin R. Grooms
}

\begin{abstract}
Context: The sensory organization test (SOT) is a standard for quantifying sensory dependence via sway-referenced conditions (sway-referenced support and sway-referenced vision [SRV]). However, the SOT is limited to expensive equipment. Thus, a practical version of the SOT is more commonly employed-the clinical test for sensory integration in balance; however, it fails to induce postural instability to the level of SRV. Objective: Determine if Stroboscopic vision (SV), characterized by intermittent visual blocking, may provide an alternative to the SRV for assessing postural stability. Design: Descriptive laboratory study. Setting: Research laboratory. Participants: Eighteen participants (9 males, 9 females; age $=22.1[2.1] \mathrm{y}$, height $=169.8[8.5] \mathrm{cm}$, weight $=66.5[10.6] \mathrm{kg}$ ). Intervention $:$ Participants completed the SOT conditions, and then repeated SOT conditions 2 and 5 with SV created by specialized eyewear. Main Outcome Measures: A repeated-measures analysis of variance was completed on the time-to-boundary metrics of center-of-pressure excursion in the anteroposterior and mediolateral directions in order to determine the difference between the full-vision, SV, and SRV conditions. Results: Postural stability with either SRV or SV was significantly worse than with full vision $(P<.05)$, with no significant difference between SV and SRV $(P>.05)$. Limits of agreement analysis revealed similar effects of SV and SRV except for unstable surface mediolateral time-to-boundary. Conclusions: In general, SV was found to induce a degree of postural instability similar to that induced by SRV, indicating that SV could be a portable and relatively inexpensive alternative for the assessment of sensory dependence and reweighting.
\end{abstract}

Keywords: balance/posture, biomechanics, instrument-assisted interventions, sensory integration

Sensory dependence is the preferential contribution of visual, vestibular, and somatosensory feedback for completing a motor task or maintaining stability and can be modified by pathologies, task conditions, or training. ${ }^{1,2}$ The sensory organization test (SOT) is the gold standard for quantifying sensory dependence by utilizing sway-referenced conditions. Specifically, the SOT engages a sway-referenced support condition to knockdown the somatosensory system and a sway-referenced vision (SRV) condition to knockdown the visual system. The SOT provides a standardized assessment of sensory contributions to postural stability and has been used extensively to evaluate concussion and fall risk and patients with balance disorders in order to inform targeted therapies for specific sensory deficits. ${ }^{1,3,4}$

However, the SOT is limited to expensive equipment; thus, the clinical test for sensory integration in balance (CTSIB) was developed. ${ }^{5}$ In the CTSIB, the sway-referenced condition of the SOT is imitated using a foam pad, and SRV is imitated using a white dome over the head. ${ }^{5}$ The foam-induced postural instability is comparable to the sway-referenced support condition, but the dome is unable to induce instability to the level of the SRV. ${ }^{6-8}$ Thus, there remains a need to develop an adequate surrogate for SRV conditions for the CTSIB.

K.-M. Kim, J.-S. Kim, and Oh are with the Department of Kinesiology and Sport Sciences, University of Miami, Miami, FL, USA. Grooms is with the Division of Athletic Training, School of Applied Health Sciences and Wellness, College of Health Sciences and Professions; and the Ohio Musculoskeletal \& Neurological Institute, Ohio University, Athens, OH, USA. Grooms (groomsd@ohio.edu) is corresponding author.
Stroboscopic vision (SV) may provide an alternative to SRV and is characterized by intermittent visual blocking via glasses or goggles. SV has recently been shown to modify visual dependence and postural instability in both stable and unstable surface conditions. ${ }^{9}$ However, a comparison to the gold standard sensory dependence for vision SRV condition has yet to be examined. If SV induces postural instability similar to that induced by SRV, then it may offer a viable option for a visually perturbed sensory dependence assessment. Thus, the purpose of this study was to compare the postural instability effects of SV against SRV on both stable and unstable surfaces. We hypothesized that both SV and SRV would significantly increase postural instability relative to full vision, but postural instability between SV and SRV would not be different and have high agreement.

\section{Methods}

Eighteen young adults (9 males, 9 females; age $=22.1[2.1] \mathrm{y}$, height $=169.8[8.5] \mathrm{cm}$, weight $=66.5[10.6] \mathrm{kg}$ ) without any history of musculoskeletal injury in the past 6 months or of vision or balance disorders participated. The University of Miami Institutional Review Board approved the study protocol requiring informed consent.

All participants completed the SOT with the NeuroCom EquiTest (Neurocom International Inc, Clackamas, OR), consistent with the testing protocol described previously. ${ }^{4}$ However, SOT conditions 2 and 5, in which participants are asked to close their eyes during testing, were replaced with SV created by specialized eyewear (Nike SPARQ Vapor Strobes; Nike Inc, Beaverton, OR) 
that intermittently cycles between opaque and transparent for 100 milliseconds at a time. ${ }^{9}$ Participants performed bipedal stances that lasted for 20 seconds and were repeated 3 times. The averages were used for statistical analysis. The study outcomes were time-toboundary (TTB) parameters of center-of-pressure excursion. We computed TTB measures such as mean and SD of the minima in the anteroposterior (AP) and mediolateral (ML) directions using previously reported methods. ${ }^{10}$ A lower TTB value reflects poorer postural stability.

For each of the TTB measures, a repeated-measures analysis of variance was performed to determine if there was a difference in postural stability on the fixed (stable) surface between 3 visual conditions as follows: (1) full vision (SOT condition 1), (2) SV (SOT condition 2), and (3) SRV (SOT condition 3). The same analysis was repeated for these conditions on the swayreferenced (unstable) surface (SOT conditions 4-6). In addition, Cohen $d$ effect sizes and associated 95\% confidence intervals (CIs) were calculated to provide the magnitude of postural instability with either SV or SRV compared to full vision using pooled SD. Cohen guidelines were used to determine the strength of the effect size as follows: (1) less than $0.2=$ trivial, (2) from 0.21 to $0.5=$ small, (3) from 0.51 to $0.8=$ moderate, and (4) greater than $0.8=$ large. ${ }^{11}$

To assess agreement between SRV and SV, we completed a one-sample $t$ test to determine difference from zero for each balance measure, Bland-Altman plots to assess agreement for each measure, and Pearson $r$ values with 95\% CIs between conditions for all measures. We also calculated $R^{2}$ values to determine the variance of SRV performance explained by SV. All statistical analyses were conducted using SPSS statistical software (version 24.0; IBM Corp, Armonk, NY) with the alpha level set a priori at $P \leq .05$.

\section{Results}

Descriptive data for the 3 visual conditions with the fixed and sway-referenced surfaces are presented in Tables 1 and 2, respectively. For postural stability on a fixed surface, there were significant differences between the 3 visual conditions for the AP mean and SD measures (Table 1). Postural stability with either SRV or SV in the AP direction was significantly worse than with full vision, but there were no significant differences between SRV and SV. The disruptive effects of SRV were small (close to moderate), and SV effects were moderate, but there was no difference in effect size between SRV and SV relative to FV, because their respective $95 \% \mathrm{CI}$ overlapped. In contrast to AP postural instability, ML stability was unaffected by SRV or SV on the stable surface.

There were significant differences for all TTB measures for postural stability on the unstable (sway-referenced) surface (Table 2). Postural stability with SRV or SV was significantly worse relative to full vision, but SV again did not significantly differ from SRV. Postural instability with SV or SRV in the AP direction was of large magnitude relative to FV. For ML postural instability, SV also had large effects relative to $\mathrm{FV}$, while SRV had small to moderate effects. Again, relative to FV, the $95 \%$ CIs of both SV and SRV overlapped, indicating no effect differences.

The limits of agreement analysis also largely supported a similar effect of SV relative to SRV. On the fixed surface, all TTB metrics had good agreement (Figure 1) and moderate to high correlation (Table $1, r=.49-.77$ ). On the unstable surface, the TTB mean and SD in the AP direction had good agreement (Figure 2), with moderate to high correlation (Table 2, $r=.65-.75)$. However, TTB mean and SD in the ML direction had condition differences greater than zero but still had high correlations (Table 2, $r=.79-.82$ ). The $R^{2}$ values for each variable between SRV and SV are reported in Tables 1 and 2 .

\section{Discussion}

We found both SRV and SV significantly impaired standing balance relative to full vision, with no significant difference between SV and SRV. Specifically, we observed small to moderate effects for AP postural instability on a stable surface, with no ML effect and moderate to large effects for AP and ML postural instability on an unstable surface with both visual conditions. The limits of agreement analysis indicated that SV and SRV induced comparable effects in all conditions for all outcome metrics except for the ML direction during the unstable surface condition. This lack of agreement may have been due to the different mechanisms of visual knockdown. The SV condition reduces visual feedback by $50 \%$ (strobe rate of $100-\mathrm{ms}$ transparent $\backslash 100$-ms opaque) and is not specific to an AP or ML corrective strategy, whereas the SRV produces inaccurate visual feedback primarily in the AP direction. Nonetheless, SV accounted for approximately $62 \%$ to $67 \%$ of the variance in SRV for ML TTB mean and SD; the remaining 33\% to $38 \%$ can potentially be attributed to differences in the ways in which visual information was manipulated. The different mechanisms of visual perturbation, while resulting in generally similar postural instability, should be considered for SOT in patients with postural instability depending on an origin of postural instability (reduced sensory feedback versus inability to recognize accurate sensory input).

Stroboscopic vision can be utilized dynamically and is portable and relatively inexpensive (\$200 or less), making it an attractive alternative for sensory reweighting assessment. The need for such a tool is especially apparent in settings dealing with highly active patients, such as in sports medicine, with emerging evidence indicating that common musculoskeletal conditions induce sensory reweighting. ${ }^{12-15}$ SOT may not be effective in athletic populations due to its reliance on a static bipedal stance not being challenging enough to discover postural instability that may be manifested during more aggressive athletic maneuvers. Thus, SV could be an alternative for progressive sensory reweighting in more active populations. Of note, our findings are limited to individuals without current injury and to the NeuroCom system. Patients, especially those with disorders affecting balance, may respond to $\mathrm{SV}$ differently; this requires future research.

\section{Conclusions}

Stroboscopic vision was found to induce a degree of postural instability similar to that induced by SRV and can be used in the assessment of sensory reweighting as part of CTSIB, allowing future investigators and clinicians to engage in more dynamic postural stability assessments. 


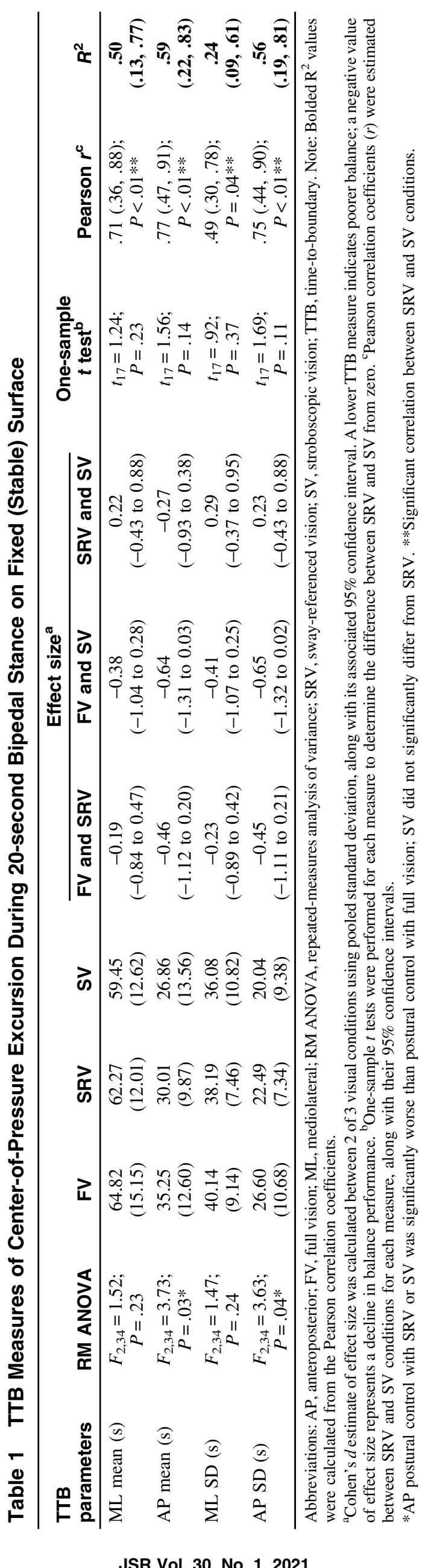




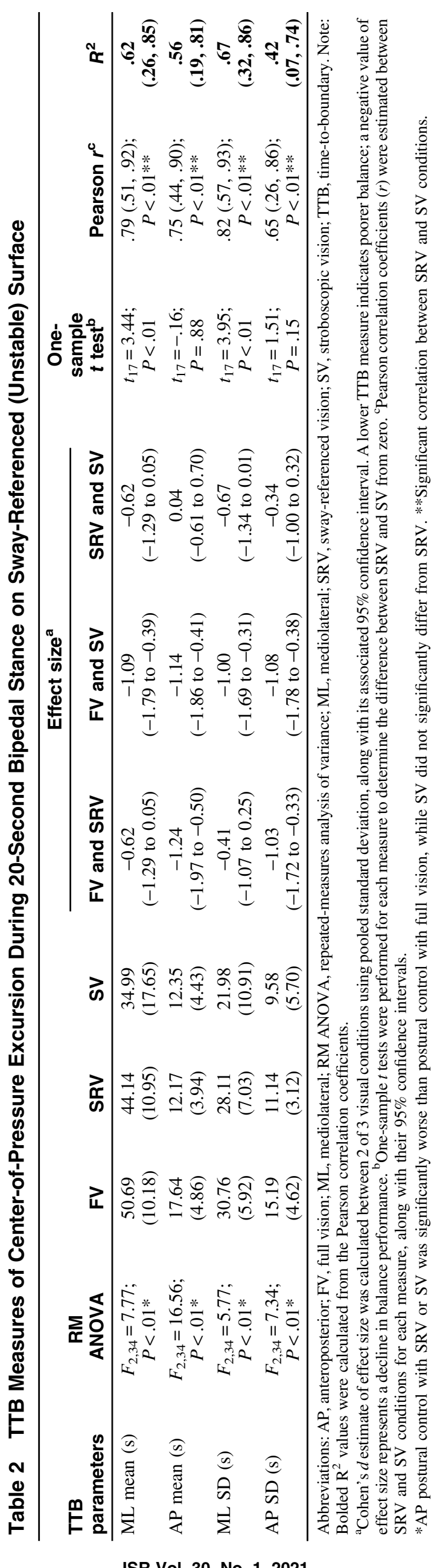




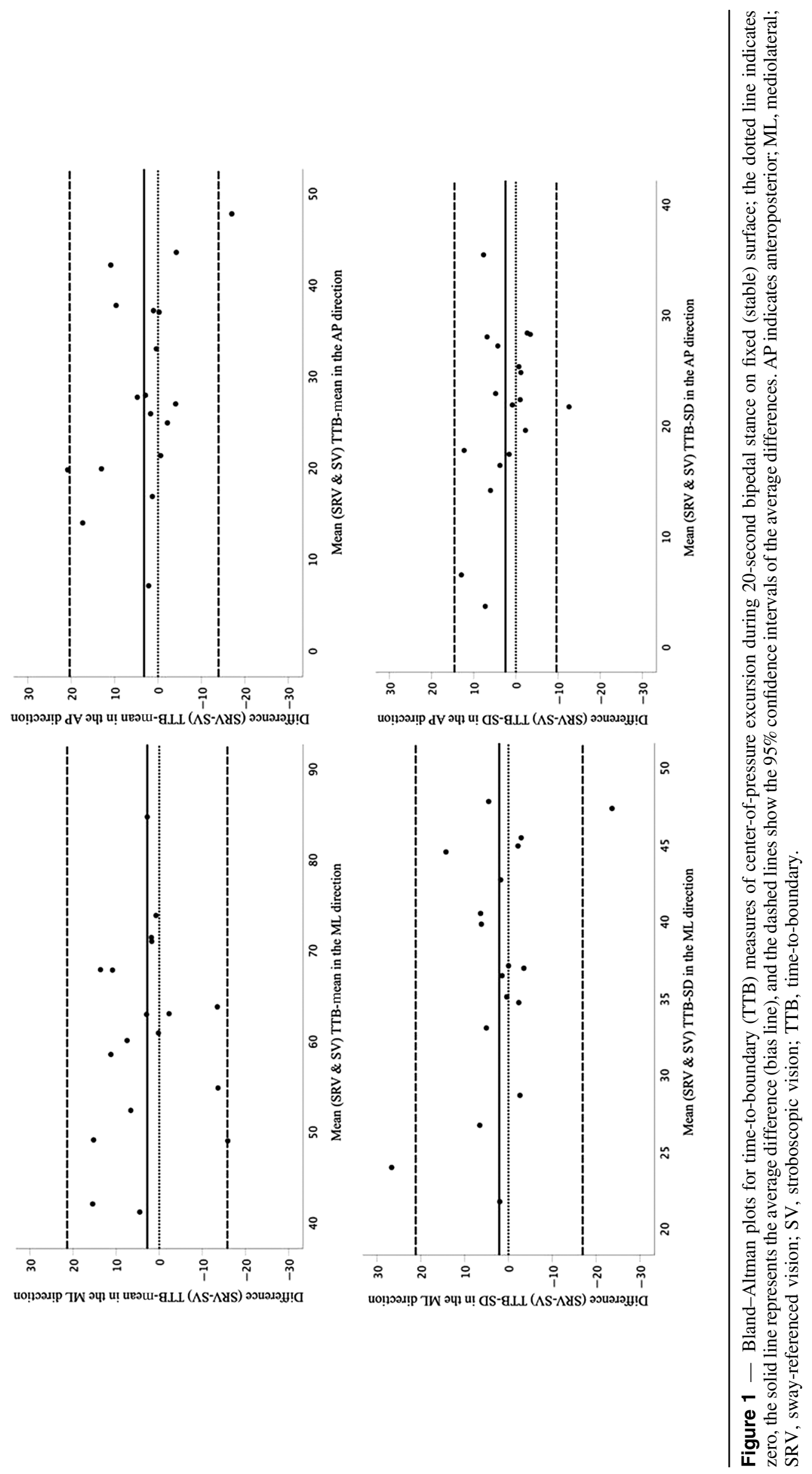




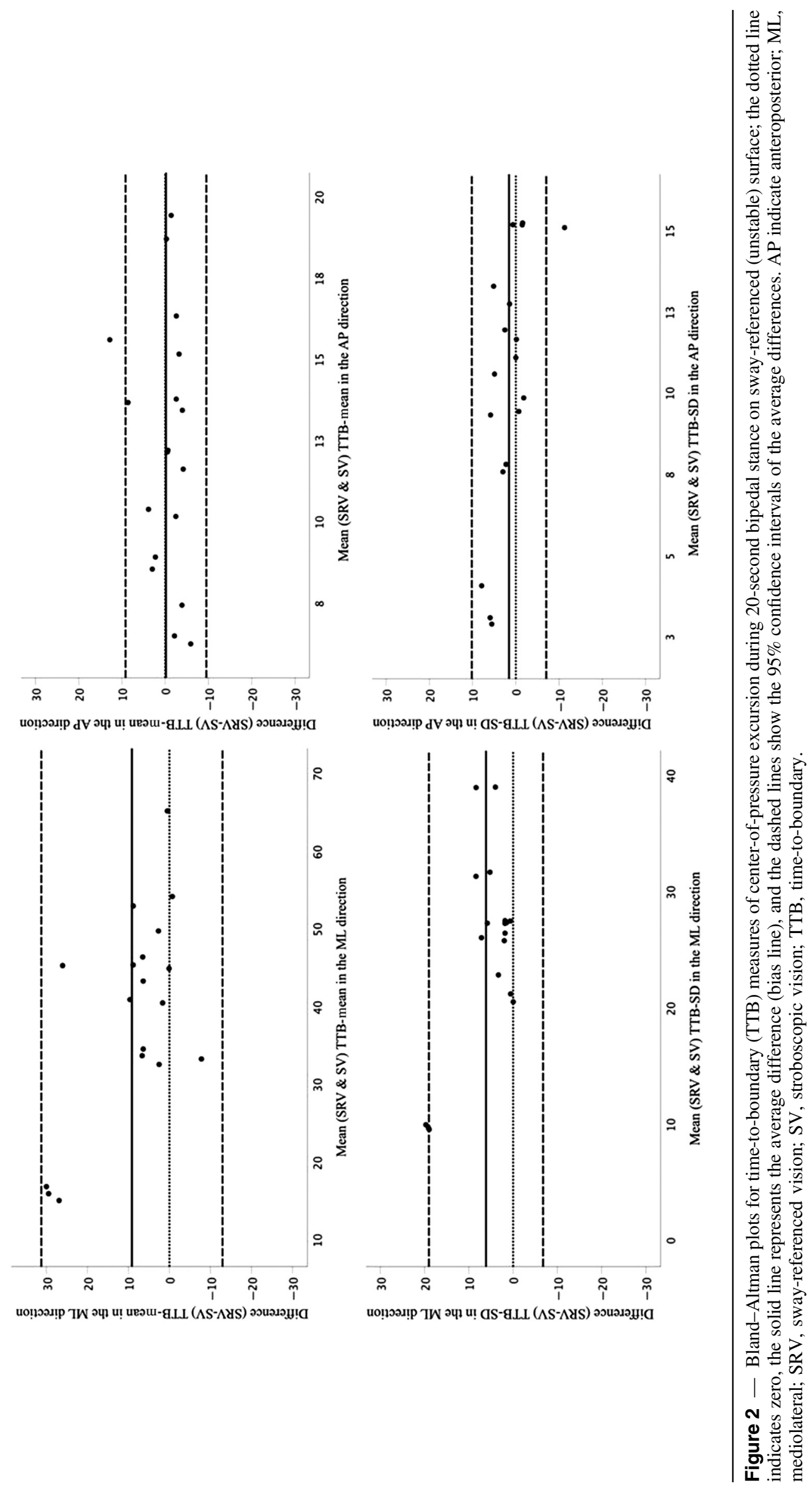




\section{Acknowledgments}

This research did not receive any specific grant from funding agencies in the public, commercial, or not-for-profit sectors. The authors have no conflicts of interest associated with this work.

\section{References}

1. Peterka RJ. Sensory integration for human balance control. Handb Clin Neurol. 2018;159:27-42. PubMed ID: 30482320 doi:10.1016/ B978-0-444-63916-5.00002-1

2. Forbes PA, Chen A, Blouin JS. Sensorimotor control of standing balance. Handb Clin Neurol. 2018;159:61-83. PubMed ID: 30482333 doi:10.1016/B978-0-444-63916-5.00004-5

3. Pierchala K, Lachowska M, Wysocki J, Morawski K, Niemczyk K. Evaluation of the Sensory Organization Test to differentiate nonfallers from single- and multi-fallers. Adv Clin Exp Med. 2019;28: 35-43. PubMed ID: 30058785 doi:10.17219/acem/76374

4. Vander Vegt CB, Register-Mihalik JK, Ford CB, Rodrigo CJ, Guskiewicz KM, Mihalik JP. Baseline concussion clinical measures are related to sensory organization and balance. Med Sci Sports Exerc. 2019;51(2):264-270. PubMed ID: 30239494 doi:10.1249/MSS. 0000000000001789

5. Shumway-Cook A, Horak FB. Assessing the influence of sensory interaction of balance. Suggestion from the field. Phys Ther. 1986; 66(10):1548-1550. PubMed ID: 3763708 doi:10.1093/ptj/66.10.1548

6. Cohen H, Blatchly CA, Gombash LL. A study of the clinical test of sensory interaction and balance. Phys Ther. 1993;73(6):346-351; discussion 51-54. PubMed ID: 8497509 doi:10.1093/ptj/73.6.346

7. Hong SK, Park JH, Kwon SY, Kim JS, Koo JW. Clinical efficacy of the Romberg test using a foam pad to identify balance problems: a comparative study with the sensory organization test. Eur Arch
Otorhinolaryngol. 2015;272(10):2741-2747. PubMed ID: 25200890 doi:10.1007/s00405-014-3273-2

8. Wrisley DM, Whitney SL. The effect of foot position on the modified clinical test of sensory interaction and balance. Arch Phys Med Rehabil. 2004;85(2):335-338. PubMed ID: 14966723 doi:10.1016/ j.apmr.2003.03.005

9. Kim KM, Kim JS, Grooms DR. Stroboscopic vision to induce sensory reweighting during postural control. J Sport Rehabil. 2017;26(5):111. PubMed ID: 28605310 doi:10.1123/jsr.2017-0035

10. Hertel J, Olmsted-Kramer LC, Challis JH. Time-to-boundary measures of postural control during single leg quiet standing. $J$ Appl Biomech. 2006;22(1):67-73. PubMed ID: 16760569 doi:10.1123/ jab.22.1.67

11. Cohen J. Statistical Power Analysis for the Behavioral Sciences. 2nd ed. Hillsdale, MI: Lawrence Erlbaum Association; 1988.

12. Song K, Burcal CJ, Hertel J, Wikstrom EA. Increased visual use in chronic ankle instability: a meta-analysis. Med Sci Sports Exerc. 2016;48(10):2046-2056. PubMed ID: 27635773 doi:10.1249/MSS. 0000000000000992

13. Wikstrom EA, Song K, Pietrosimone BG, Blackburn JT, Padua DA. Visual utilization during postural control in anterior cruciate ligament- deficient and -reconstructed patients: systematic reviews and meta-analyses. Arch Phys Med Rehabil. 2017;98(10):2052-2065. PubMed ID: 28483655 doi:10.1016/j.apmr.2017.04.010

14. Claeys K, Brumagne S, Dankaerts W, Kiers H, Janssens L. Decreased variability in postural control strategies in young people with nonspecific low back pain is associated with altered proprioceptive reweighting. Eur J Appl Physiol. 2011;111(1):115-123. PubMed ID: 20824281 doi:10.1007/s00421-010-1637-x

15. Kim KM, Kim JS, Oh J, Lee SY. Time-to-boundary analysis of postural control following acute lateral ankle sprain. Gait Posture. 2019;67:151153. PubMed ID: 30340127 doi:10.1016/j.gaitpost.2018.10.002 